\title{
Microneurography in relation to intraneural topography: somatotopic organisation of median nerve fascicles in humans
}

\author{
Rolf G Hallin
}

\begin{abstract}
Microneurography was performed in median nerve sensory fascicles with concentric needle electrodes and with conventional tungsten microneedles. The latter electrodes preferentially recorded activity from the myelinated fibres in the whole fascicle. By contrast, due to its special design, a concentric needle can record activity selectively from even a small part of a fascicle. High amplitude signals in $\mathrm{C}$ fibres can be discriminated close to Schwann cells that envelope unmyelinated axons. Apart from being biased for activity in thin fibres, the concentric needles can also record signals from nearby myelinated fibres. The palmar receptive fields of such fibre groups were not congruent with the areas traditionally attributed to multiunit skin afferents in humans, namely the innervation zone(s) of one or two adjacent digital nerve(s). Instead, the multiunit fields often comprised small parts of a digital nerve innervation area, frequently only the pulp of a finger. Single units were always localised within previously screened multiunit areas. Contrary to some previously accepted tenets it is probable that single unit activity in myelinated fibres in these studies is recorded extra-axonally near to a node of Ranvier. The findings also suggest the presence of a somatotopy in human limb nerve fascicles, comparable to that previously established in the spinal cord and the somatosensory cortex.
\end{abstract}

By using surface electrodes, Eichler succeeded in recording synchronous mass activity through the skin from a mixed nerve in situ. The subsequent use of improved macroelectrodes placed in the vicinity of a peripheral nerve, ${ }^{2-4}$ later combined with averaging procedures, refined the technique of recording activity from intact limb nerves in humans. ${ }^{45}$ These procedures have been used for both experimental and clinical purposes. ${ }^{4-7}$

Single unit activity in humans was first recorded experimentally from excised and/or exposed nerves in situ, an approach which obviously is precluded from wide adoption in humans. ${ }^{8}$ Vallbo and Hagbarth described a percutaneous technique to explore human limb nerves with intraneurally positioned solid tungsten needle microelectrodes. 'This technique records multiunit and single unit activity in myelinated fibres. ${ }^{9}$ Multiunit activity in $\mathrm{C}$ fibres innervating muscle ${ }^{10}$ and skin, ${ }^{11} 12$ as well as single unit activity in both afferent and sympathetic $C$ fibres, ${ }^{13-15}$ can also be discerned.

Hallin and Wiesenfeld introduced a standardised, thin diameter concentric needle electrode for percutaneous recording of $\mathrm{A}$ and C fibre units in humans. ${ }^{16}$ In this investigation the recording properties of the concentric needle electrode were compared with those of the tungsten needle. Further, individual median nerve fascicles were screened for their relative contents of myelinated and unmyelinated fibres and to obtain information about the cutaneous palmar distribution of these fibres. Preliminary data ${ }^{16}{ }^{17}$ and our results indicate that the concentric electrode is biased towards recording $\mathrm{C}$ fibre activity. The data obtained also suggest the presence of a hitherto not described somatotopic organisation in human sensory nerve fascicles.

\section{Material and methods}

Our findings were obtained from 67 experiments performed on 15 healthy subjects of both sexes aged between $20-42$ years. They all gave their informed consent to participate in the trials. In eight experimental sessions skin nerve activity was recorded from the median nerve at the wrist and in 49 sessions from the nerve just proximal to the elbow. For comparison ten experiments with tungsten electrodes were carried out under equivalent conditions.

\section{A Recording electrodes}

In most experiments concentric needle electrodes were used. ${ }^{16}$ They consisted of commercially available hypodermic needles with outer shaft diameters of 200-250 $\mu \mathrm{m}$ and inner diameters of $90-100 \mu \mathrm{m}$ (fig 1 ). A thin insulated tungsten wire, diameter $10-30 \mu \mathrm{m}$ or a thin platinum-iridium wire of diameter 20 $30 \mu \mathrm{m}$ was positioned inside the needle, which was then filled with Araldite. The leads from the central core and the shaft (ground lead) were thin copper wires. The impedance of electrodes with small diameter recording wires $(10 \mu \mathrm{m})$ was $500-700 \mathrm{k} \Omega$, and of those with the largest threads $(30 \mu \mathrm{m}) 200-300 \mathrm{k} \Omega$ at $1000 \mathrm{~Hz} \cdot{ }^{18}$ Solid tungsten electrodes (bare tip length $30-100 \mu \mathrm{m}$, impedance $50-75 \mathrm{k} \Omega$ ) were used in a few experiments. ${ }^{91920}$ 


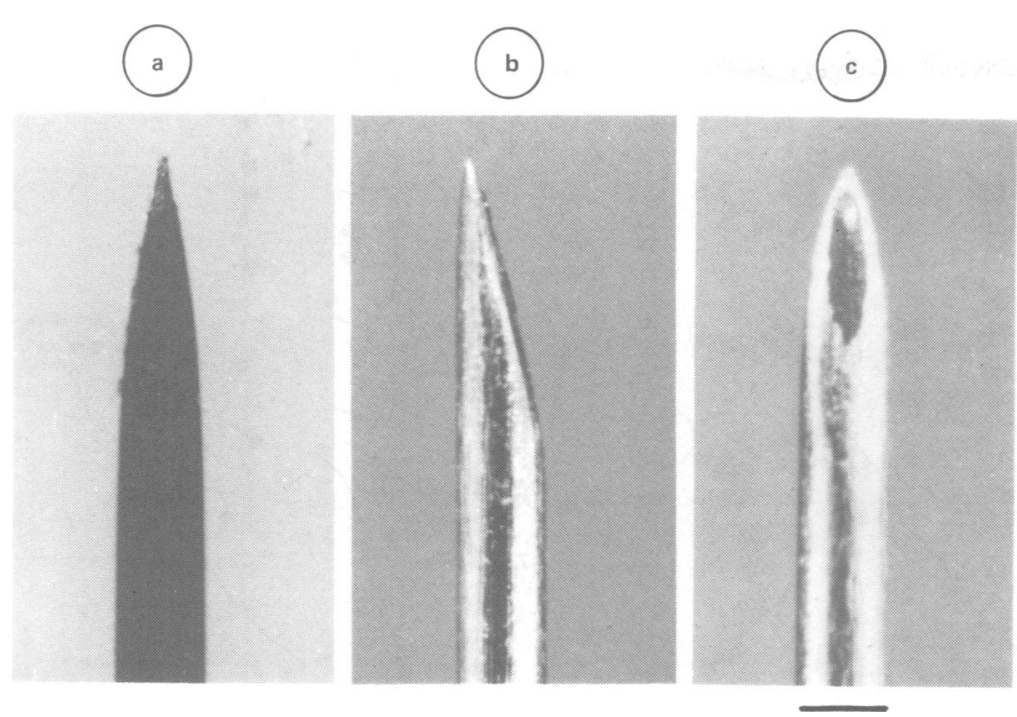

Figure 1 Photomicrographs of a tungsten wire microelectrode and its tip region $(A)$ and similar photographs of a concentric needle electrode, as described in the text, in profile $(B)$ and with its recording surface in full view $(C)$. The sizes of the two types of electrodes are similar. The length of the tip of this tungsten electrode was $135 \mu \mathrm{m}$ (side of cone formed by the tip $140 \mu \mathrm{m}$, base $67 \mu \mathrm{m}$ ) and the size of its conical recording area was estimated as $14200 \mu^{2}$. The recording surface of this concentric electrode was $20 \times 50$ $\mu \mathrm{m}$ as estimated in the microscope and its oval recording area amounted to $785 \mu \mathrm{m}$. (The measures of the recording area of the concentric electrode appear overemphasised due to an unproportionally strong light reflex in the metal surface.) Magnification $\times 38$. Bar $200 \mu \mathrm{m}$.

The flat oval recording areas of the concentric electrodes were much smaller than the conical recording surfaces of the tungsten needles. The recording area of the concentric electrode in fig 1C was calculated to $785 \mu \mathrm{m}^{2}$. With a core diameter of $30 \mu \mathrm{m}$ the maximal recording areas of these electrodes were about $2100 \mu \mathrm{m}^{2}$. The somewhat asymmetrical mantle area of the tungsten electrode shown for comparison in fig $1 \mathrm{~A}$ was calculated to about $14200 \mu \mathrm{m}^{2}$. If the tip of such a tungsten electrode had extended only 70 respectively $100 \mu \mathrm{m}$, the base of the cone would have been about $28 \mu \mathrm{m}$ respectively $38 \mu \mathrm{m}$ and the recording areas would have been about 3100 $\mu \mathrm{m}^{2}$ respectively $6000 \mu \mathrm{m}^{2} .^{20}$

\section{$B \quad$ Recording and display system}

The recording electrodes were connected to commercially available preamplifiers (Neurolog System, Digitimer Ltd). For maximal recording safety the ground was close to the recording point/intraneural stimulating site. The safety measures taken corresponded to those of class I, group B. The bandwidth of the whole system was generally $200 \mathrm{~Hz}-10 \mathrm{KHz}(-40 \mathrm{~dB} /$ decade), but it could be modified when needed by applying other filter settings. An improvement to the signal-to-noise ratio was obtainable by an amplitude discriminator which could eliminate about $50 \%$ of the noise. ${ }^{19}$ The noise of the recording system peak-to-peak was about 10$15 \mu \mathrm{V}$. The best units had amplitudes of 40 $60 \mu \mathrm{V}$. During the experiments the signals were displayed on an oscilloscope, fed into a loudspeaker and were simultaneously stored on tape.

\section{Mechanical and thermal stimuli}

The receptive field location and extent of low threshold mechanoreceptors was mapped by a number of stimuli, such as application of skin taps or pressure with various small objects. The thresholds of individual units and boundaries of the unitary receptive fields were examined by using von Frey's hairs. Afferent C fibre activity was also evoked by squeezing or applying pressure to the skin, needle pricks in the receptive area or by applying warm or hot stimuli derived from a radiant heat source (Quartslampen $\mathrm{GmbH}$, Original Hanaan, West Germany) or from a commercially available temperature stimulator (Somedic AB, Stockholm, Sweden) operating according to the Peltier principle. ${ }^{21}$

\section{Electrical stimulation}

Electrical stimulation (square wave pulses of $0 \cdot 1-1$ ms duration) was performed using a DISA stimulator unit (Type 14E D1). Surface or needle electrodes were used for skin stimulation. Intraneural electrical stimulation was performed with the recording area of the electrode as cathode and the shaft of the needle as anode.

\section{E Nerve exploration procedure}

The experiments were carried out as described previously. ${ }^{16}{ }^{19}$ To minimise the risks of inducing nerve fibre damage the bevelled surface of the concentric needle was directed through the skin in parallel with and into the nerve to be explored. Weak electrical pulses (1-6 V, $0.2 \mathrm{~ms}$ duration) intraneurally eliciting paraesthesias in the fascicle territory, were delivered through the electrode during the search procedure to guide the electrode tip into the nerve. A number of criteria were used to decide whether the electrode picked up activity from fascicles destined for skin or muscle. ${ }^{919}$ Occasionally it was tedious to reach and keep a recording position but sometimes the nerve activity had stable amplitudes allowing studies for up to several hours.

\section{PRECAUTIONS}

The electrodes were sterilised in $35 \%$ formaline vapour at $80^{\circ} \mathrm{C}$ at a variable underpressure. The skin at the electrode site was carefully cleaned with pure alcohol and then with sterile injection swabs. The same recording site was not explored again until 4-6 weeks after an examination. With these measures no enduring neurological symptoms were encountered in any of the subjects.

\section{Results}

NERVE TOPOGRAPHY IN RELATION TO RECORDING SITES

\section{Extrafascicular sites}

The concentric electrode could be gently pushed stepwise through the nerve without causing too much discomfort to the subject. ${ }^{19}$ Electrical silence on a number of tests suggested that the recording surface often was located in extrafascicular sites. Judging from the frequency of occurrence of such situations a substantial portion of human peripheral nerve is made up of non neural tissue elements. 223 

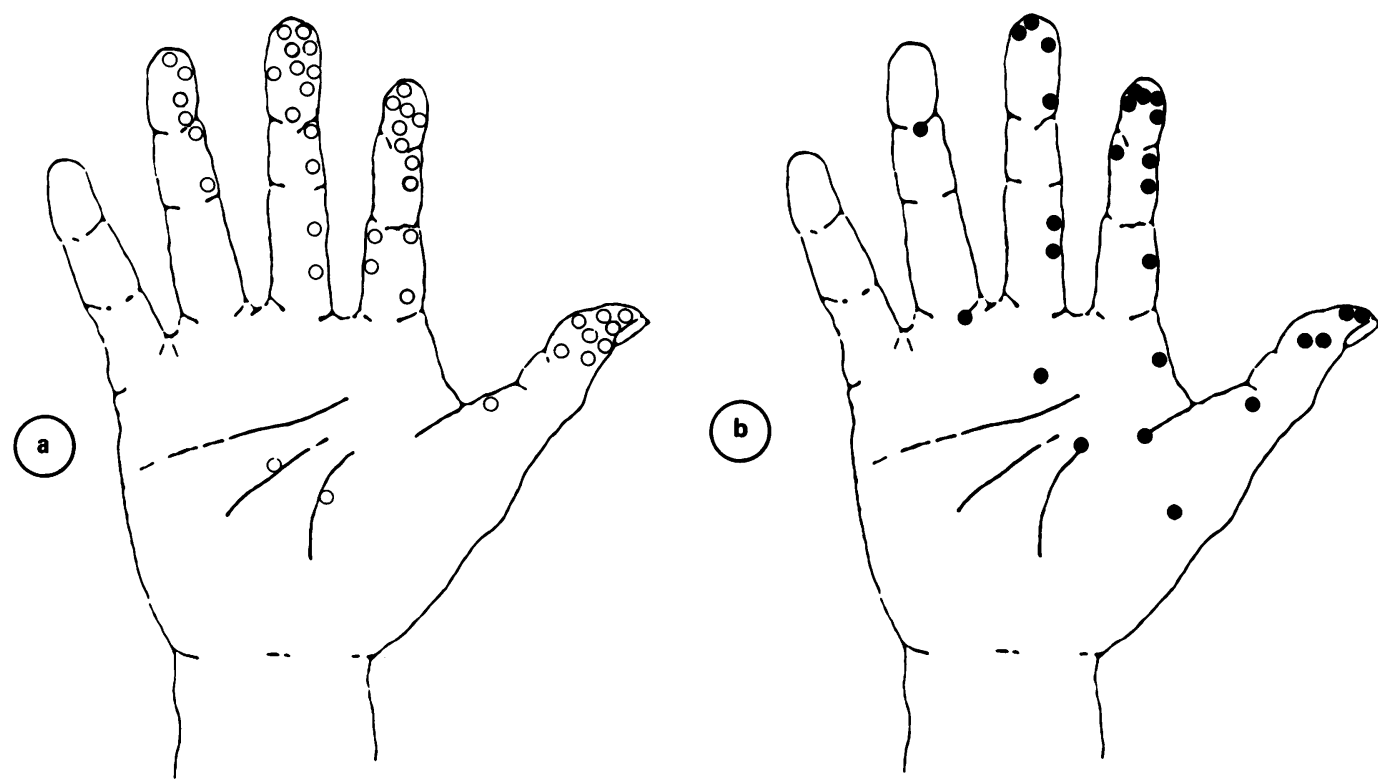

Figure 2 Anatomical distribution of receptive fields for $R A(A)$ and $S A I$ units $(B)$. The circles and dots mark receptive field locations, not actual field sizes in this figure and in fig 4. In this figure and figs 4 and 6-7 all receptive fields are displayed on diagrams of the right hand.

\section{Intrafascicular sites dominated by $A$ fibre} activity

\section{Single unit activity}

When the needle was in a stable intrafascicular site single unit activity in myelinated fibres was regularly identified either directly or after repeated needle adjustments. The occurrence of single unit activity in the recording was sometimes preceded by the appearance of injury discharges. Injury activity often occurs before unit activity is reached with tungsten microelectrodes. ${ }^{919}$ The characteristics of stable single A fibre units agreed well with those previously described in microneurography. ${ }^{194-26}$ Out of a total of 82 units $42 \mathrm{RA}$ (rapidly adapting) units, 5 PC (Pacinian afferents), 27 SAI (slowly adapting type I) and 4 SAII (slowly adapting type II) units were reliably identified (figs 2,3 ) on the basis of action potential amplitude and shape, distribution and size of receptive fields, firing characteristics etc. Four units could not be classified satisfactorily. The localisations of the units' receptive fields (fig 2) were essentially the same as previously reported when using tungsten microelectrodes for sampling ${ }^{24}$ with a high concentration of the RA and SAI units on the tip of the digits and a lower density in the rest of the hand. The sizes of their receptive fields were also as previously reported. Furthermore, the extent and sensitivity of an individual unit's receptive area remained the same during an experiment and no tendency to "blocking" of even a part of a unitary receptive field was seen. ${ }^{27}$ Representative recording sequences illustrating the firing characteristics of these units are shown in fig 3. Various details in the firing characteristics of single units were sometimes possible to test over several hours.

Generally only one (core diameter $10 \mu \mathrm{m}$ ) or a few units (1-3) (core diameter 20-30 $\mu \mathrm{m}$ ) were recorded at each site even at elbow level.
By contrast, tungsten electrodes may in a single site discriminate several units in the median nerve, especially at the elbow. The RA and SAI units were most commonly identified in recording sites where the corresponding multiunit receptive field(s) involved the pulp and an adjacent small skin region at the

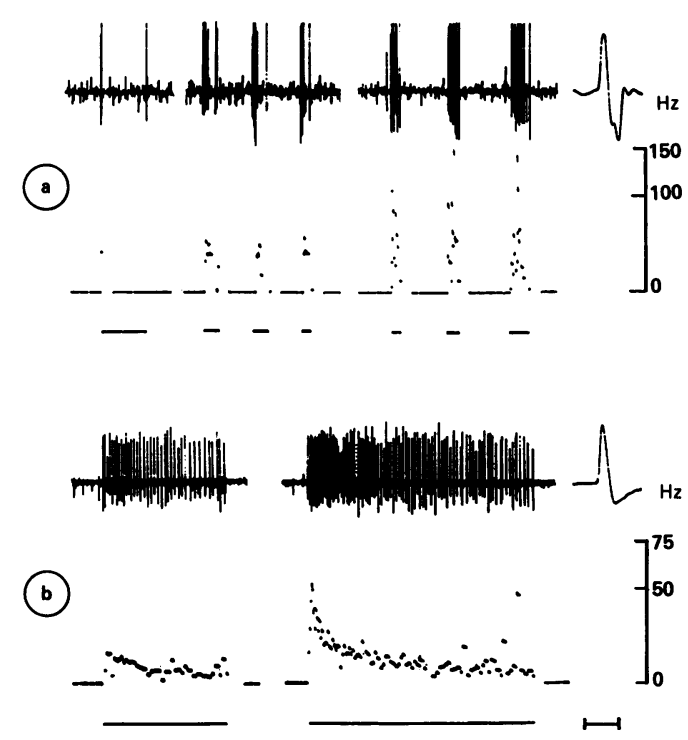

Figure 3 Discharge pattern and instantaneous firing frequency of a RA unit localised in glabrous skin of the third finger tip $(A)$ and a SAI unit with receptive field in the pulp of the index finger $(B)$. Time bar for $A-B 1 \mathrm{~s}$, for impulses of respective unit to the right $1 \mathrm{~ms}$.

$A$ : The $R A$ unit was phasic and responded with only one or a few spikes at the application and removal of a constant pressure of $0.6 \mathrm{~g}$ (bar) exerted with a von Frey hair (left). Brisk strokings along the dermal ridges (bars) induced brief bursts with an instantaneous firing of 60-90 $\mathrm{Hz}$ (middle). In response to equally brisk strokings across the dermal ridges the unitary firing rose to $150 \mathrm{~Hz}$ (right).

$B:$ The illustrated SAI unit fired in a typical manner with an initial burst at the application of the stimulus and then slowly adapting in a graded manner depending upon the stimulus strength (4.1 g to the left, $9 \cdot 2 \mathrm{~g}$ to the right) as long as the pressure was maintained (bars). 

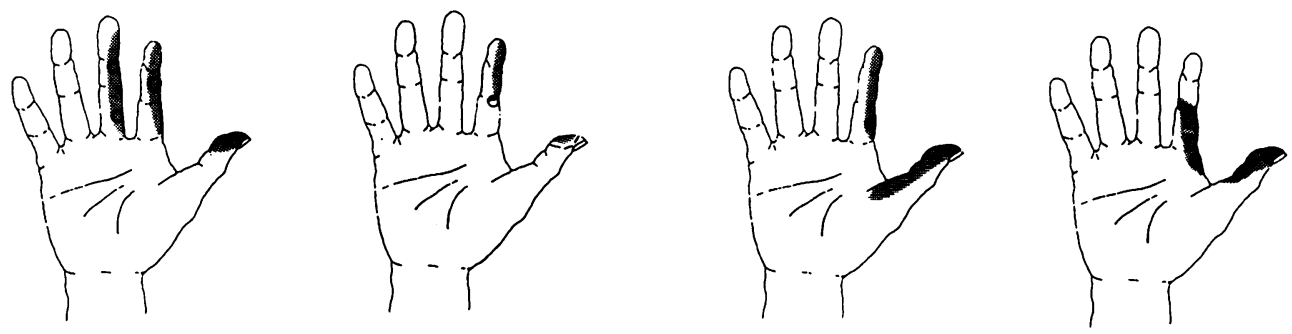

(a)
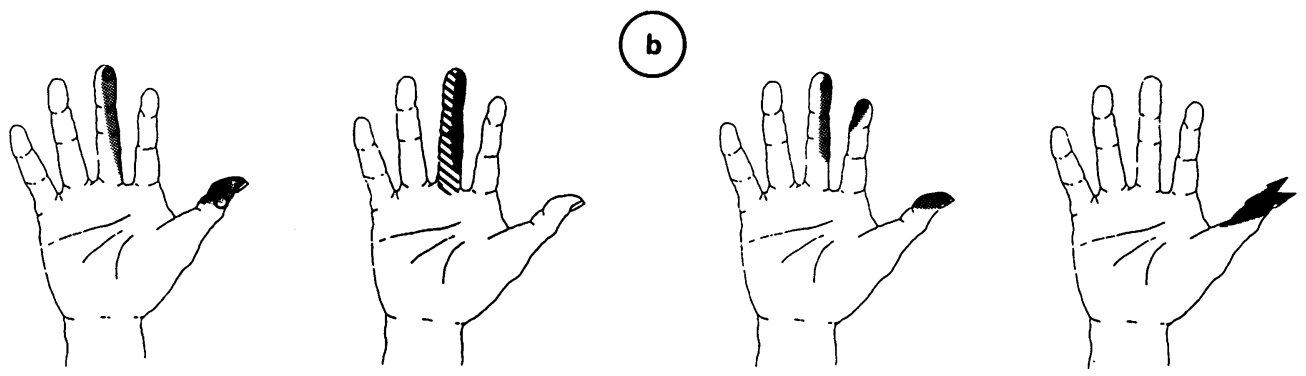

Figure 4 Relationship between identified single unit receptive sites and corresponding multiunit receptive fields. $R A$ and $P C$ receptor sites are shown in $A, S A I$ and $S A I I$ sites in $B$. The units were either identified when the multiunit area was initially mapped or encountered in the course of a few needle adjustments when abandoning the original recording site. The hatched areas display the established extent of the receptive fields for PC and SAII receptors and the black dot the point of maximal sensitivity of these receptors.

$A$ : The $R A$ units (circles) were always localised within the previously established multiunit field (grey area) irrespective of field size and complexity. The outcome of five different experiments are shown to the left. To the right above an example of a split multiunit receptive field comprising a relatively large part of the pulp of the thumb (maximal extension of not visible area indicated by broken lines) and part of the index finger is shown. Below to the right is displayed the field of one $P C$ receptor.

$B$ : Seven SAI receptor sites (dots) and one SAII site (below right) obtained from different experiments and their relation to the corresponding multiunit receptive fields are shown. Moderate skin tension in the directions indicated by the arrows enhanced the firing of the SAII unit.

finger tip (see below). When sampling tactile afferents in such a situation by repositioning the electrode intraneurally, the units were always encountered within or sometimes on the border of the receptive field for the previously recorded multiunit activity (fig 4) which was reduced in amplitude when a large amplitude single unit was discriminated. These findings were consistent irrespective of recording site and irrespective of whether the previously identified multiunit field was small, large or split (figs $4,6,7$ ). Sometimes successive units were of the same modality and had adjacent receptive fields. In one recording at the elbow four units, three RA units and one SAI unit were found in a cluster in the pulp of the thumb (fig $4 \mathrm{~A}$, bottom row left).

At the end of the experiments the electrode was sometimes successively withdrawn from the recording site in the nerve. During this procedure previously identified units tended to reappear in the neurogram in the reverse order to that in which they had been identified.

\section{Multiunit activity}

The multiunit activity evoked by weak miechanical stimuli appeared as low amplitude, mainly negative directional mass discharges. The most intense activity was evoked by brief repeated stimulation with, for example, rough emery cloth or vibratory stimuli (fig $5 \mathrm{~A}$ ). Responses occurred at the onset and offset of local pressure whereas often only relatively sparse discharges were recorded during maintained stimulation. The multiunit discharges recorded with the concentric needles often had considerably lower amplitudes than such activity recorded with tungsten needles (5C, D). In some cases, however, and particularly when the activity was derived from pulp afferents, well discriminated responses to both local touch stimuli and maintained pressure were discerned, especially in the integrated neurogram (fig 5A, B).

The receptive field locations and distribution in the hand were as described earlier. ${ }^{19}$ However, the multiunit areas recorded with the concentric needle electrodes were usually smaller $\left(50-70^{\circ}{ }_{0}\right.$ less) than those normally found with the tungsten needles (figs 4,6 ). When exploring the median nerve at the wrist the receptive area generally included an elongated, slightly asymmetrical part of a finger tip and a coherent small region on the phalanx next to the pulp. Sometimes the innervation area was more proximal and then involved glabrous skin of the two proximal phalanges on the ulnar or radial aspect of the finger (fig 6A). Similar size multiunit receptive fields were found at the elbow level, although larger receptive areas were also found there, corresponding to the innervation area of one or two or sometimes parts of two adjacent digital nerves (fig 6B). In almost all experiments the position, extension and complexity of the multiunit receptive fields of low threshold skin afferents changed slightly when repositioning the electrode surface in the nerve fascicle in small steps. This finding was consistent irrespective of whether the recording site was at the wrist or at the elbow level (fig 7). 


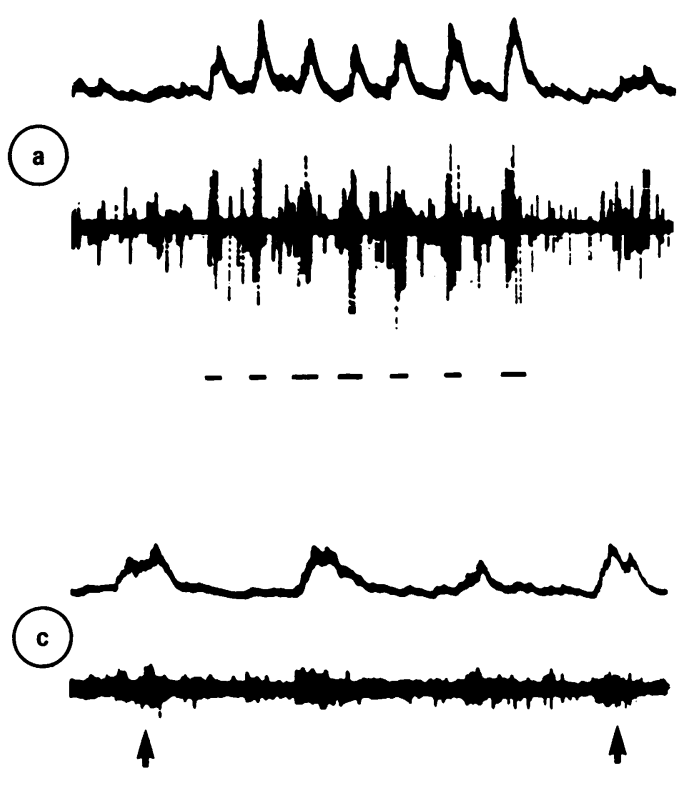

(b)
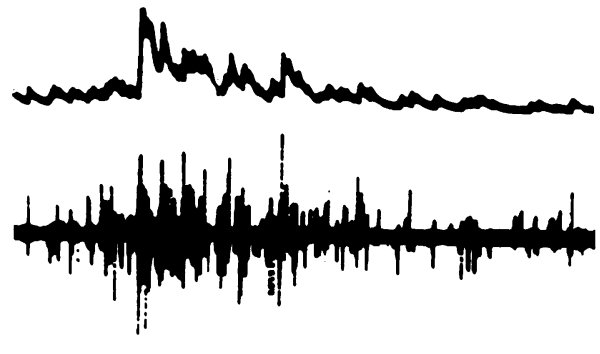

(d)

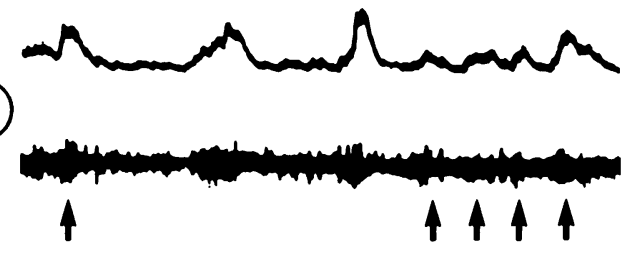

Figure 5 Integrated neurograms (above) and multiunit responses (below) to repeated strokings of the skin with an emery cloth (left, bars) and to a continuously applied constant pressure with a metal springholder (right, bar). Time constant of integrater $500 \mathrm{~ms}$. Time calibration $1 \mathrm{~s}$. A, B: The afferent multiunit discharges were sometimes well discriminated. The phasic component of these responses appeared most distinctly. $C, D$ : Different recording site than $A-B$. The multiunit receptive field was split and comprised the pulp region and the skin covering the ulnar part of the first phalanx of the thumb and the basal radial part of the index finger on the left hand. This recording was dominated by sympathetic activity. The afferent multiunit discharges to skin strokings and constant pressure in the thumb were discrete but sometimes the amplitudes of the responses were as high as that of spontaneous outbursts of sympathetic skin nerve activity (arrows).

Intrafascicular sites dominated by C fibre activity In many experiments the initial recording was that of $C$ fibre activity, generally as sympathetic multiunit discharges. This was less common with tungsten than concentric needles, especially when exploring the median nerve.

The general occurrence of the sympathetic bursts during resting conditions and under a variety of manoeuvres, such as cooling or stressing the subject, fully agreed with previously described characteristics of cutaneous sympathetic outflow in humans. ${ }^{28}{ }^{29}$ The sympathetic multiunit discharges often had higher amplitudes than concomitantly recorded A fibre multiunit activity (fig 5) and the corresponding receptive area for myelinated fibres on many occasions was positioned proximally in the fingers and palm. Slight needle adjustments occasionally dramatically improved the signal-to-noise-ratio in such a recording so that single unit activity in sympathetic fibres could be recorded.

Afferent $C$ fibre activity was recorded on several occasions, but was not, like sympathetic activity, encountered at every recording site. The activity was in many instances of a unitary character already when first identified and the signal-to-noise-ratio of the discharges could sometimes be improved on needle adjustment. In most recording sites about two or three $C$ afferents were identified. Only exceptionally was an afferent $C$ unit recorded in isolation. Additional data on $\mathrm{C}$ fibre activity will be published separately. ${ }^{30}$

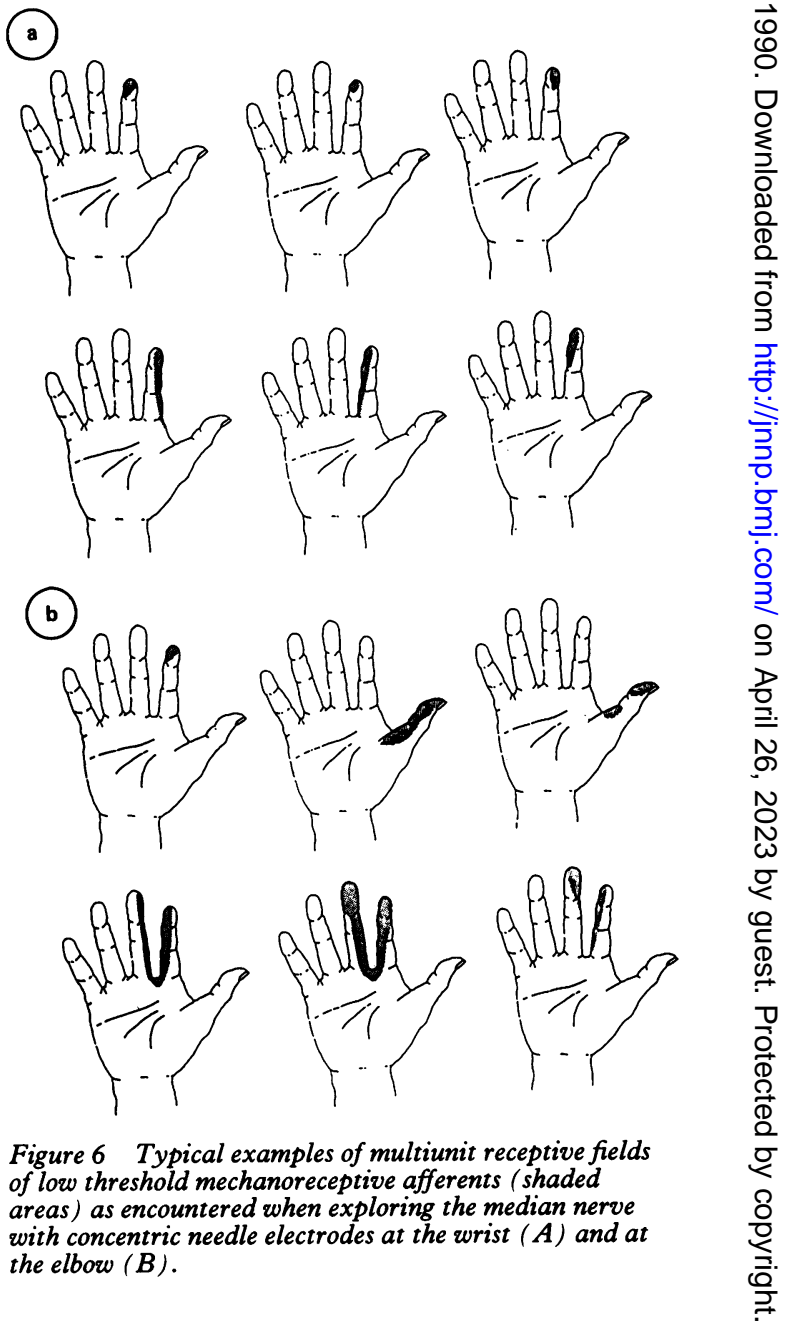


$\odot$
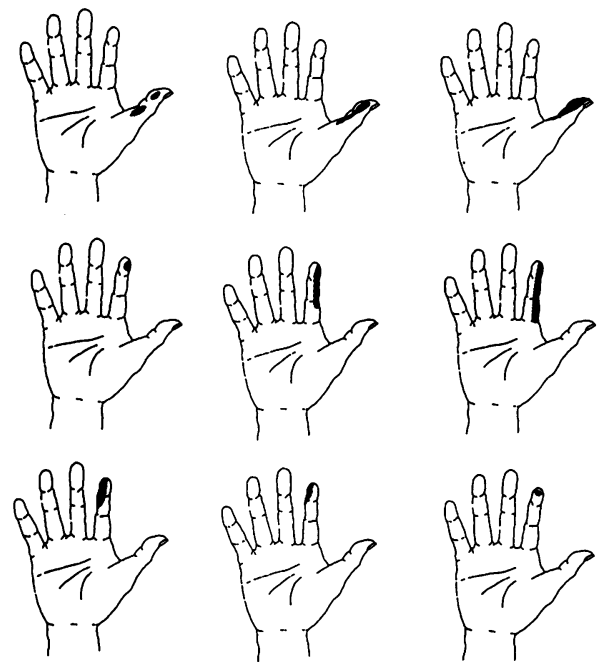

2

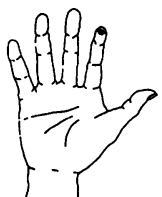

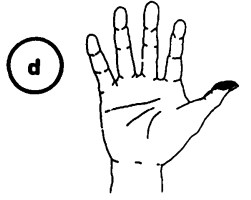
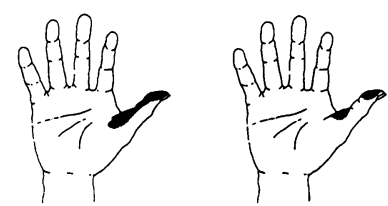

(-)
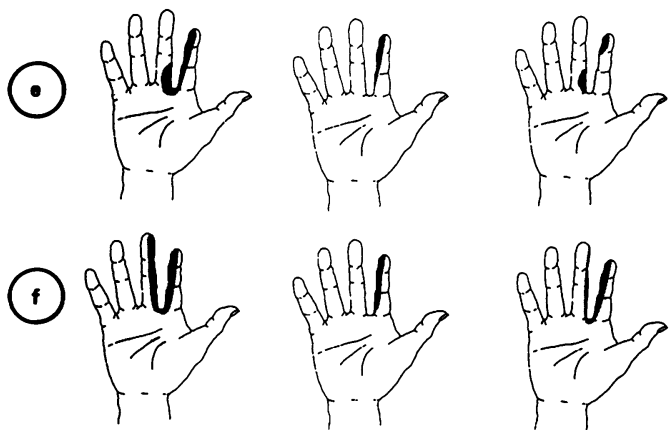

2

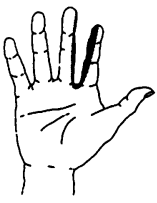

3

Figure 7 Transition of localisation and extent of multiunit receptive fields (shaded areas) in successive intraneural sites (1-3) when mapping the median nerve at the wrist $(A-C)$ and at the elbow $(D-F)$. Both at the wrist (three experiments) and at the elbow (three experiments) changes in the multiunit receptive field were noted upon minute adjustments of the concentric recording needle (1-3) irrespective of whether the needle was advanced into $(A, D, E)$ or withdrawn from the nerve $(B, C, F)$.

\section{Discussion}

Possible hazards involved in the exploring procedures which might influence the interpretation of the results

Electrodes inserted into human nerve fascicles might mechanically or otherwise interfere with and distort the intraneural environment so that neurophysiological events become misrepresented in the recorded activity. Wall and McMahon ${ }^{27}$ paid particular attention to this problem and suggested that single unit recordings from human peripheral nerves only are possible because almost all of the nerve fibres near the electrode tip are pressure blocked during the exploring procedure and therefore unable to conduct action potentials. Only one or a few fibres near the tip would stay active, making action potentials recordable as unitary discharges. ${ }^{27}$

In a study utilising concentric needle electrodes with two recording surfaces we established the probability of recording neural activity at both surfaces at the same time. ${ }^{31}$ In $80 \%$ of the experiments nerve signals were discerned at both recording areas simultaneously. Furthermore, the properties of the identified units to a diversity of stimuli were similar to characteristics ascribed to normal, previously established receptor types in subhuman species..$^{32-36}$ Therefore, gross functional abnormalities engaging the explored nerve fascicle do not seem to be prevailing during microneurographic investigations.

\section{Electrophysiology related to intraneural topography. Impulse generators for single and multiunit $A$ and $C$ fibre activity}

As pointed out in a critical review unitary discharges in microneurography are not likely to be recorded intra-axonally. ${ }^{27}$ Furthermore, unit activity in myelinated fibres recorded with the concentric needle can hardly originate from an intra-axonally positioned electrode surface. On the other hand, the suggestion of a pressure block for most nerve fibres at the electrode site, thereby making unit identification possible, could not be verified. ${ }^{31}$

Another unconsidered possibility to account for how unit activity can be recorded in these studies would be that the unitary signals in the case of myelinated fibres represent activity at the nodes of Ranvier. This does not exclude the possibility that the tip of a tungsten electrode recording unit activity may occasionally be partially penetrating the axon. ${ }^{37-39}$ As will be discussed, the present data would be easily accounted for if the proposed idea of unit origin is accepted. Furthermore, some major discrepancies between the present results and previous microneurographic data acquired with the tungsten electrode could then be explained.

Concentric electrodes with small recording surfaces seldom recorded single unit activity in myelinated fibres whereas electrodes with somewhat larger surfaces did so regularly, sometimes from several units. These data fitted well with our experience that sampling of units with myelinated fibres tended to be more rewarding when using tungsten electrodes (unpublished observations). Obviously, the big three dimensional tip area of such an electrode will record from a larger number of nodes of Ranvier than the concentric needles used (fig 8).

The unitary characteristics, for example, shape and duration of the recorded potentials and firing pattern to various stimuli were virtually the same irrespective of recording technique. However, injury discharges were more rare with the concentric needle and the identified units (fig 3 ) in many cases showed no overt signs of nerve fibre damage. ${ }^{173}$ In this context it should be recalled that previously identified but abandoned unit(s) tended to reappear in focus for the electrode when it was moved out of the nerve. The likelihood for such events to occur would be much higher if the unit recordings originated near some anatomical structure(s) than if relocation of the recording surface intra-axonally in one or more previously penetrated axons would be neces- 


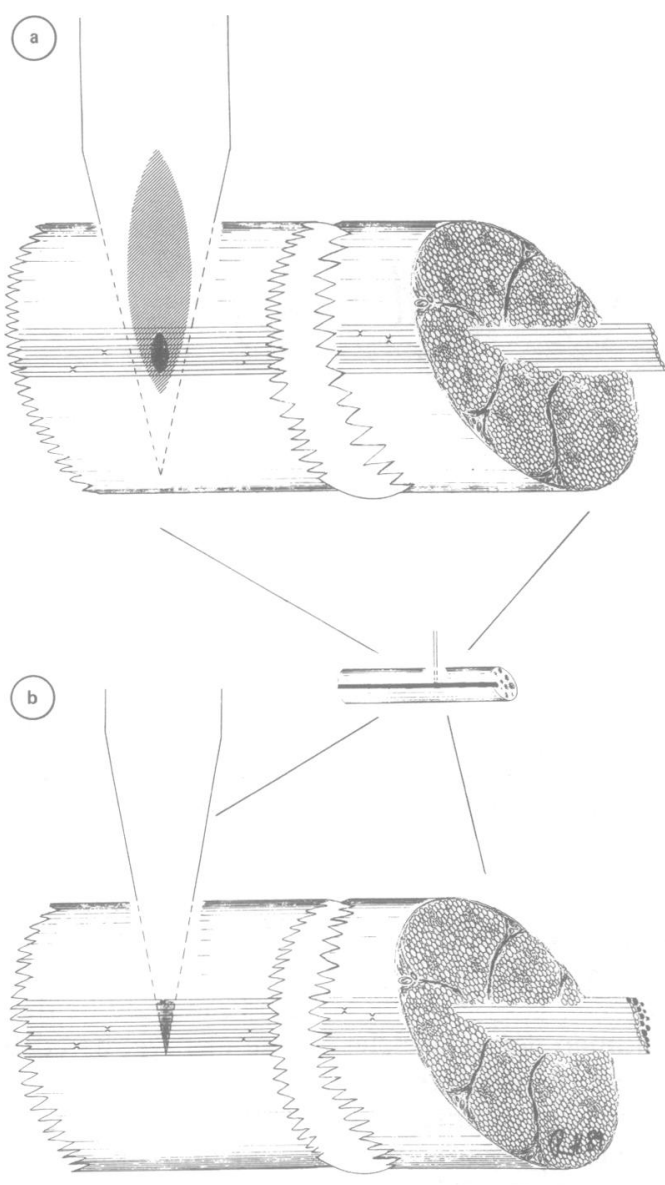

Figure 8 Schematic illustration of the proposed recording site when using the concentric needle $(A)$ and the tungsten electrode $(B)$ for microneurography. The small illustration in the middle indicates that a recording electrode inserted in a nerve has entered into a fascicle. In $A$ is shown how 10 fibres lie near to the recording surface (shaded area measuring about $30 \times 85 \mu \mathrm{m}$ ) of the concentric electrode (hatched oval indicates the Araldite filled lumen of the needle). Sometimes a node of Ranvier may lie close to the recording area resulting in a unit recording. Many more fibres (20) lie close to the recording area of a tungsten electrode (length of free tip 'shaded area) in this case $100 \mu \mathrm{m}$ ) in the same recording site (B). Apart from a few more fibres lying in front of the needle, most of the additional fibres (indicated by black ellipses to the right) are located "behind" the needle. (Their course behind the needle is not indicated.) Addititional nodes of Ranvier may get close to such a needle with its conical recording area resulting in additional unit recordings. It is also apparent that the concentric needle records multiunit activity preferentially from fibres close to and in front of its recording surface. The tungsten electrode records multiunit activity from fibres abutting on any part of its bare conical tip and will therefore reflect the overall activity from a large part or the entirety of the explored fascicle. Bar $100 \mu \mathrm{m}$.

sary for unit recording.

Thus it is suggested that unitary activity in myelinated fibres sampled during microneurography is recorded extra-axonally near a node of Ranvier. In the nodal-paranodal part of the membrane the number of ion channels is maximal. ${ }^{40}$ Furthermore, the generation of action currents is concentrated to the node. ${ }^{41} 42$ At the node, high amplitude signals from a thick fibre would therefore be expected, provided that the two closely spaced electrode surfaces of the concentric needle are close to the active site. ${ }^{43}$ Away from the nodal region the longitudinal current density outside the fibre is negligibly small, ${ }^{41}{ }^{42}$ making single unit recordings from a myelinated fibre substantially less likely at the internodes. Also, the shape of the unitary impulses were of the types to be expected when recording the electrical activity from a fibre very close to a node of Ranvier with the kind of electrodes used in this investigation (Frankenhaeuser, personal communication).

Multiunit A (discussed under next heading) and $\mathrm{C}$ fibre signals probably represented extracellular activity recorded close to a number of fibres, but distant from a node. Since the intervals between the nodes may amount to several hundred microns, ${ }^{44}{ }^{45}$ which is long in relation to the length of the electrode surface, and the nodes are not always randomly distributed within the fascicle (Hallin and Ekedahl, unpublished observations), the location of the active surface of the needle will often be at the internodes. Solely multiunit activity therefore should (and does) occur in the recordings quite frequently during microneurography. The receptive fields were smaller than those found using solid tungsten electrodes for nerve exploration ${ }^{19}$ and the evoked multiunit mechanoreceptor activity from these fields was often of very low amplitudes. Reasons were given above to explain why the concentric needles used in this investigation often sampled fewer units in a recording site than tungsten needles. The same line of argument could explain why the multiunit mechanoreceptor activity often had lower amplitudes and regularly derived from smaller receptive fields when screened with the concentric needle than when the tungsten electrode was used for similar nerve explorations (fig 8).

Multiunit C activity was often better discriminated than multiunit A fibre discharges with the present technique (fig 5). This finding would also relate to the design of the concentric needle. Since it often records activity from only a small part of the myelinated fibres of the fascicle, multiunit discharges in A fibres were less prominent than they would have been if recorded by a tungsten electrode in the same site (fig 8). Thus simultaneously recorded C fibre activity tended to be favourably enhanced, especially close to the electrode when exploring nerve fascicles with the concentric needle.

\section{Somatotopy in human median nerve fascicles}

A number of mapping experiments were performed to study intraneural fascicular topography with the more selective concentric needle. In many trials the multiunit fields of myelinated afferents comprised a part or the entire pulp of a finger (figs $4,6,7$ ). In about $30 \%$ of the cases the fields were split in at least two distinct areas, suggesting simultaneous recordings from more than one nerve fibre population. Following adjustments of the electrode position there were minor changes in the extent and localisation of the multiunit innervation area (fig 7). There were also striking transitions from fields composed by separate parts to fields which were coherent and vice versa. The single units, however, which were found in the course of the changes in recording site were all situated within the boundaries of the previously screened multiunit receptive fields (fig 4 ) and in one experiment several single A fibre elements 
(three RA and one SAI unit) with separate but nearby receptive areas were successively identified in a cluster in the pulp of the thumb. These findings provide strong evidence for the presence of intrafascicularly localised microbundles of myelinated fibres in human nerves. Some microbundles seem to be destined to the finger pulp, others to more proximal parts on the side of a finger. Furthermore, cutaneous median nerve fascicles at the elbow level, which typically contain fibres supplying the innervation areas of two adjacent digital nerves, probably contain at least four (fig 7), probably more, bundles of myelinated fibres which innervate adjacent sides and pulp regions of two neighbouring fingers.

In contrast to our results, Schady et al found that the myelinated fibres in human peripheral nerve fascicles are randomly distributed intrafascicularly..$^{20}$ The issue then is whether or not the interpretation of our data can also account for the discrepancies in the results between the two studies. Due to its design, the concentric electrode is quite useful in analysing the neural activity from small groups of fibres close to the recording surface (fig $8 \mathrm{~A}$ ). Since the tungsten microelectrode discriminates activity in many more fibres around its entire conical tip (fig $8 \mathrm{~B}$ ) it is simply not possible by physiological recordings to study microanatomy reliably in small portions of the fascicle by using this technique. Hence, the now discovered fascicular organisation was not detected in previous microneurographic recordings with tungsten electrodes. ${ }^{19}$ Neither microstimulation through the intrafascicularly inserted tungsten electrode, ${ }^{39}$ used by Schady et al to study intrafascicular fibre organisation in detail, ${ }^{20}$ seems accurate enough in the present context. In the trials described it was assumed that individual nodes of Ranvier within small groups of fibres close to the electrode tip were stimulated. However, since the recording/ stimulating tungsten electrode sometimes had a free tip extending as much as $180 \mu \mathrm{m},{ }^{20}$ which presumably was totally enclosed intrafascicularly, and there was no way to judge the distribution of the stimulating currents used, the nodes of Ranvier of the stimulated fibres near such a big tip might well have been spread out within a comparatively large intrafascicular volume. Intraneural stimulation of several dispersed nodes of Ranvier near the whole free tip would evoke randomly scattered projected fields, indicative of unit activation, within a large cutaneous area within the explored fascicular territory, thus accounting for the reported results. ${ }^{20}$ However, in view of the present data it is apparent that the microstimulation technique is not sufficiently precise to use as a tool for studying the intrafascicular fibre organisation in detail. ${ }^{30}$

Thus we conclude that an orderly microsomatotopy is present in human median nerve fascicles. The results adhere to a common principle of a somatotopical organisation in the somatosensory system which starts in the peripheral nerves and relays via the spinal cord $^{46}$ to end in a well organised manner at more central levels. ${ }^{47}$
Some clinical implications

The fingers in primates have a dense mechanoreceptive innervation. ${ }^{245}$ The intrafascicular grouping of the myelinated fibres supplying palmar skin must also have bearing on tactile gnosis in humans. Thus the relatively common early complaint of finger tip numbness occurring in patients with carpal tunnel syndrome might be accounted for as a compression involving microbundles destined to the finger tips. If neighbouring median nerve fibres destined to the whole hand and the finger pulps were randomly distributed in each individual fascicle as suggested in another study ${ }^{20}$ the distal numbness occurring in the carpal tunnel syndrome would be much less likely to develop especially if the massive central representation of the fingers is considered. Clinically, reality points in the opposite direction.

Our results may also be of relevance for corrective micro-surgery aiming to re-establish innervation after nerve trauma. It is well known that the manual discriminatory capacity restored after peripheral nerve suture to a substantial part depends on the extent of the nerve injury. Patients with partial nerve lesions generally regain a functionally more complete tactile gnosis than those with total severance of the nerve ${ }^{48}$ which is easily understood in the light of the present findings.

Judging from the number of recording sites where sympathetic activity occurred, sympathetic fibres are quite numerous in the median nerve. ${ }^{30}$ This is not surprising as the unmyelinated fibres far outnumber myelinated fibres in human nerves, ${ }^{4950}$ but quite the contrary to the experience with the tungsten microelectrode which records preferentially from the myelinated fibre population of the whole fascicle. ${ }^{1924}$ The fact that sympathetic fibres are numerous in the median nerve might be one necessary prerequisite for the occurrence of pain syndromes involving the median nerve area. ${ }^{51}$

I am grateful to Professor Bernhard Frankenhaeuser for valuable discussion of the manuscript. The expert secretarial aid of Maj-Len Holm and Eli Fröyen-Stenberg is gratefully acknowledged. This research was supported by funds at the Karolinska Institute and The Swedish Society of Medicine.

1 Eichler W. Über die Ableitung der Aktionspotentiale vom menschlichen Nerven in situ. $Z$ Biol 1937;98:182-214.

2 Dawson GD, Scott JW. The recording of nerve action potentials through skin in man. $J$ Neurol Neurosurg Psychiatry 1949;12:259-67.

3 Dawson GD. The relative excitability and conduction velocity of sensory and motor nerve fibres in man. $J$ Physiol 1956;131:436-51.

4 Buchthal F, Rosenfalck A. Evoked action potentials and conduction velocity in human sensory nerves. Brain Res 1966;3:1-122.

5 Gilliat RW, Mellville JD, Velate AS, Willison RG. A study of normal nerve action potentials using an averaging of normal nerve action potentials using an averaging
technique (Barrier grid storage tube). J Neurol Neurosurg
Psychiatry 1965;28:191-200.

Psychiatry 1965;28:191-200.
6 McLeod JG. Digital nerve conduction in the carpal tunnel syndrome after mechanical stimulation of the finger. $J$
symed syndrome after mechanical stimulation of
Neurol Neurosurg Psychiatry 1966;29:12-22.

7 Sears TA. Action potentials evoked in digital nerves by stimulation of mechanoreceptors in the human finger. $J$ Physiol 1959;148:30-31.

8 Hensel H, Boman KKA. Afferent impulses in cutaneous sensory nerves in human subjects. $J$ Neurophysiol 1960;23:564-78. 
9 Vallbo AB, Hagbarth KE. Activity from skin mechanoreceptors recorded percutaneously in awake human subjects. Exp Neurol 1968;21:270-89.

10 Hagbarth KE, Vallbo ÅB. Pulse and respiratory grouping of sympathetic impulses in human muscle nerves. Acta Physiol Scand 1968;74:96-108.

11 Hallin RG, Torebjörk HE. C-fibre components in electrically evoked compound potentials recorded from human median nerve fascicles in situ. Acta Soc Med Upsalien 1970;75:77-80.

12 Hallin RG, Torebjörk HE. Electrically induced A and C fibre responses in intact human skin nerves. Exp Brain Res 1973;16:309-20.

13 Hallin RG, Torebjörk HE. Afferent and efferent $C$ units recorded from human skin nerves in situ. Acta Soc Med Upsalien 1970b;75:277-81.

14 Torebjörk HE, Hallin RG. C-fibre units recorded from human sensory nerve fascicles in situ. Acta Soc Med Upsalien 1970;75:81-4

15 Torebjörk HE, Hallin RG. Identification of afferent $C$ units in intact human skin nerves. Brain Res 1974;67:387-403.

16 Hallin RG, Wiesenfeld Z. A standardized electrode for percutaneous recording of $A$ and $C$ fibre units in conscious man. Acta Physiol Scand 1981;113:561-3.

17 Wiesenfeld-Hallin Z, Hallin RG. The influence of the sympathetic system on mechanoreception and nociception. A review. Human Neurobiol 1984;3:41-6.

18 Jahnke MT. Properties of a microcoaxial electrode designed for unit recording from human peripheral nerves. $J$ Ner unit recording from human pethods 1982;6:335-46.

19 Hagbarth KE, Hongell A, Hallin RG, Torebjörk HE. Afferent impulses in median nerve fascicles evoked by tactile stim $423-42$.

20 Schady WJL, Torebjörk HE, Ochoa JC. Peripheral projections of nerve fibres in the human median nerve. Brain Res 1983;277:249-61.

21 Kenshalo DR. Improved method for the psychophysical study of the temperature sense. Review of Scientific Instruments 1963;34:883-6.

22 Sunderland $S$. The intraneural topography of the radial, median and ulnar nerves. Brain 1945;68:243-99.

23 Sunderland S, Bedbrook GM. The cross-sectional area of peripheral nerve trunks occupied by the fibres representperipheral nerve trunks occupied by the findual muscular and cutaneous branches. Brain ing individual 4 .

24 Johansson RS, Vallbo AB. Tactile sensory coding in the glabrous skin of the human hand. Trends Neurosciences glabrous skin

25 Knibestöl M. Stimulus-response functions of rapidly adapting mechanoreceptors in the human glabrous skin area. $J$ Physiol 1973;232:427-52.

26 Knibestöl $M$. Stimulus-response functions of slowly adapting mechanoreceptors in the human glabrous skin area. $J$ Physiol 1975;245:63-80.

27 Wall PD, McMahon SB. Microneurography and its relation to perceived sensation. A critical review. Pain 1985; 21:209-29.

28 Delius W, Hagbarth KE, Hongell A, Wallin BG. Manoeuvres affecting sympathetic outflow in human skin oeuvres affecting sympathetic outflow in

29 Hagbarth KE, Hallin RG, Hongell A, Torebjörk HE, Wallin BG. General characteristics of sympathetic activity in human skin nerves. Acta Physiol Scand 1972;84:164-76.

30 Hallin RG, Ekedahl R, Frank O. Segregation by modality of myelinated and unmyelinated fibres in human sensory nerve fascicles. Muscle Nerve (in press).
31 Hallin RG, Wiesenfeld-Hallin Z, Duranti R. Percutaneous microneurography in man does not cause pressure block of Letters 1986;68:356-61.

32 Iggo A. An electrophysiological analysis of afferent fibres in primate skin. Acta Neuroveg (Wien) 1962;24:225-40

33 Iggo A. Cutaneous receptors with a high sensitivity to mechanical displacement. In: de Reuck AWS, Knight J, eds. Ciba Symposium Touch, Heat and Pain. London: Churchill, 1966:237-56.

34 Mountcastle VB, Talbot WH, Kornhuber HH. The neural transformation of mechanical stimuli delivered to the monkey's hand. In: de Reuck AWS, Knight J, eds. Ciba Symposium Touch, Heat and Pain. London: Churchill, 1966:325-45.

35 Talbot WH, Darian-Smith I, Kornhuber HH, Mountcastle VB. The sense of flutter-vibration: Comparison of the human capacity with response patterns of mechanorecephuman capacity with response patterns of mechanoreceptive afferents

36 Burgess PR, Perl ER. Cutaneous mechanoreceptors and nociceptors. In: Iggo A, ed. Handbook of Sensory Physiology, Vol II. Berlin: Springer Verlag, 1973:29-78.

37 Tasaki I. Properties of myelinated fibres in frog sciatic nerve and in spinal cord as examined with microelectrodes. Jap J Physiol 1952;3:73-94.

38 Torebjörk HE, Hallin RG, Hongell A, Hagbarth KE. Single unit potentials with complex waveform seen in microelectrode recordings from the human median nerve. Brain Res 1970;24:443-50

39 Ochoa J, Torebjörk HE. Sensations evoked by intraneural microstimulation of single mechanoreceptor units
innervating the human hand. $J$ Physiol 1983;342:633-54.

40 Röper J, Schwarz KR. Heterogeneous distribution of fast and slow potassium channels in myelinated rat nerve fibres. J Physiol 1989;416:93-110

41 Frankenhaeuser B. Saltatory conduction in myelinated nerve fibres. J Physiol 1952;118:107-12.

42 Huxley AF, Stämpfli R. Evidence for saltatory conduction in peripheral myelinated nerve fibres. J Physiol 1949; 108:315-39.

43 Adrian ED, Bronk DW. The discharge of impulses in motor nerve fibres. Part II. The frequency of discharge in reflex and voluntary contractions. J Physiol 1929;67:119-51.

44 Friede RK, Meier T, Diem M. How is the exact length of an internode determined? J Neurol Sciences 1981;50:217-28.

45 Tuczinski HJ, Friede RL. Internodal length in ventral roots of bovine spinal nerves varies independently of fibre calibre. J Anat 1984;138:423-33.

46 Kausz M, Rethelyi M. Lamellar arrangement of neuronal somata in the dorsal root ganglion in the cat. Somatosensory Research, Vol 2, no 3. 1985:193-204.

47 Dykes RW, Rasmusson DD, Hoeltzell RB. Organization of the primary somatosensory cortex in the cat. J Neurophysiol 1980;43:1527-46.

48 Grabb WC, Bement SL, Koepke GH, Green RA. Comparison of methods of peripheral nerve suturing in monkeys. Plastic and Reconstructive Surgery 1970;46:31-38.

49 Ranson SW, Drogemueller WH, Davenport HK, Fischer C. Number, size and myelination of the sensory fibers in the Number, size and myelination of the sensory fibers in the cerebrospinal

50 Tomasch J, Britton WA. On the individual variability of fibre composition in human peripheral nerves. J Anatomy 1956;90:337-49.

51 Bonica JJ. The Management of Pain. Philadelphia: Lea and Febiger, 1954. 